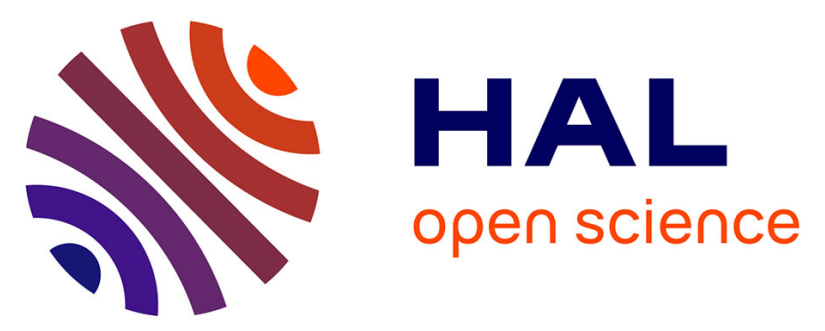

\title{
Efficacité et avenir de l'aérosolthérapie dans le traitement des infections à Pseudomonas aeruginosa chez les patients atteints de mucoviscidose
}

Amélie Rognon, Christophe Curti, Marc Montana, Thierry Terme, Pascal Rathelot, Patrice Vanelle

\section{To cite this version:}

Amélie Rognon, Christophe Curti, Marc Montana, Thierry Terme, Pascal Rathelot, et al.. Efficacité et avenir de l'aérosolthérapie dans le traitement des infections à Pseudomonas aeruginosa chez les patients atteints de mucoviscidose. Thérapie, 2011, 66, pp.481 - 491. 10.2515/therapie/2011074 . hal-01425563

\section{HAL Id: hal-01425563 \\ https://hal.science/hal-01425563}

Submitted on 3 Jan 2017

HAL is a multi-disciplinary open access archive for the deposit and dissemination of scientific research documents, whether they are published or not. The documents may come from teaching and research institutions in France or abroad, or from public or private research centers.
L'archive ouverte pluridisciplinaire HAL, est destinée au dépôt et à la diffusion de documents scientifiques de niveau recherche, publiés ou non, émanant des établissements d'enseignement et de recherche français ou étrangers, des laboratoires publics ou privés. 


\title{
Efficacité et avenir de l'aérosolthérapie dans le traitement des infections à Pseudomonas aeruginosa chez les patients atteints de mucoviscidose
}

\author{
Amélie Rognon ${ }^{1}$, Christophe Curti ${ }^{1,2}$, Marc Montana ${ }^{2}$, Thierry Terme ${ }^{2}$, Pascal Rathelot ${ }^{1,2}$ et Patrice Vanelle ${ }^{1,2}$ \\ 1 Service Central de la Qualité et de l'Information Pharmaceutique, Assistance Publique des Hôpitaux de Marseille, Marseille, France \\ 2 Laboratoire de Pharmaco-Chimie Radicalaire (LPCR), Faculté de Pharmacie, Universités d'Aix-Marseille I, II, et III - CNRS, UMR 6264, \\ Laboratoire Chimie Provence, Marseille, France
}

Texte reçu le 31 janvier 2011 ; accepté le 10 juin 2011

\section{Keywords:} cystic fibrosis; Pseudomonas aeruginosa; aerosol therapy

\begin{abstract}
Mots clés : mucoviscidose ; Pseudomonas aeruginosa ; aérosolthérapie

Résumé - Pseudomonas aeruginosa est considéré comme l'agent pathogène le plus redoutable chez les patients atteints de mucoviscidose. Son éradication est une priorité afin d'éviter le passage à l'infection chronique, véritable tournant de la maladie. Pour ceci, un large panel thérapeutique d'antibiotiques intraveineux existe, et depuis quelques années, la recherche s'intéresse de plus en plus à la voie inhalée. La synthèse des données de la littérature ici présentée se focalise à la fois sur des molécules déjà éprouvées (colistine et tobramycine), tout en présentant des thérapeutiques récentes ou à venir. Cette revue a pour but de dégager les avantages et inconvénients de chacune de ces options thérapeutiques, tout en mettant en évidence la nécessité d'études complémentaires afin de réellement prouver les intérêts thérapeutiques du panel de molécules en développement.
\end{abstract}

\begin{abstract}
Efficacy and Future of the Aerosoltherapy in the Treatment of Cystic Fibrosis Patients Infected by Pseudomonas aeruginosa. Pseudomonas aeruginosa is considered as the most redoubtable pathogenic agent at cystic fibrosis patients. Its eradication is a priority to avoid the passage to chronic infection, the real turning point of the disease. For this, a wide therapeutic panel of intravenous antibiotics exists, and for some years, the research teams concentrate more and more on the inhaled way. The synthesis of the literature data presented herein focuses on both already experienced molecules (colistin and tobramycin), and on new therapeutics. This review aims at loosening advantages and inconveniences of each of these therapeutic options, while bringing to light the necessity of follow-up studies in order to prove the therapeutic interests of molecules in development.
\end{abstract}

\section{Introduction}

La mucoviscidose est une maladie héréditaire à transmission autosomique récessive très fréquemment retrouvée dans la population occidentale. En France, son incidence a été récemment estimée à 1 naissance sur 4700, et sa prévalence à 0,75/10 000 habitants. ${ }^{[1]}$ L'atteinte pulmonaire de la mucoviscidose conduit à l'apparition d'une dilatation des bronches compliquée d'une infection bactérienne chronique. Environ $80 \%$ des patients atteints de mucoviscidose développent une infection à Pseudomonas aeruginosa $(P A)$ qui est un indicateur fortement corrélé au taux de mortalité dans la mucoviscidose. ${ }^{[2,3]}$ Les antibiotiques disponibles font de plus l'objet de résistances croissantes, souvent associées à une observance et une tolérance mauvaises avec une efficacité moindre. En 2003, une conférence de consensus sur la prise en charge du patient atteint de mucoviscidose a permis d'établir des recommandations de bonne pratique sur la stratégie de l'antibiothérapie, quelle que soit la voie d'administration, vis-à-vis de $P A$ chez le patient atteint de mucoviscidose (tableau I). ${ }^{[4]}$

Ces dernières années, les stratégies thérapeutiques ont évolué et se sont essentiellement focalisées sur l'aérosolthérapie. Bien que l'éradication de la bactérie soit impossible au stade 
Tableau I. Recommandations dans la prise en charge d'infections à $P A$ chez le patient atteint de mucoviscidose. D'après ${ }^{[4]}$

\begin{tabular}{|c|c|c|}
\hline \multirow[t]{2}{*}{ Primocolonisation } & & $\begin{array}{l}\text { Association de } 2 \text { antibiotiques bactéricides par IV pendant } 14 \text { à } 21 \mathrm{j} \text { : } \\
\beta \text {-lactamines (ceftazidime) + aminosides (tobramycine), suivi ou non } \\
\text { d'aérosol de colistine pendant } 3 \text { à } 6 \text { mois. }\end{array}$ \\
\hline & & $\begin{array}{l}\text { Le protocole Danois non validé, mais proposé comme alternative : } \\
\text { association de ciprofloxacine per os et d'aérosol de colistine. }\end{array}$ \\
\hline \multirow[t]{2}{*}{ Infection Chronique } & $\begin{array}{l}\text { Traitement des } \\
\text { exacerbations }\end{array}$ & $\begin{array}{l}\text { Bithérapie } \beta \text {-lactamines }+ \text { aminosides (tobramycine). } \\
\text { Trithérapie si souches multirésistantes. } \\
\text { Aérosolthérapie non justifiée. }\end{array}$ \\
\hline & $\begin{array}{l}\text { Traitement } \\
\text { systématique } \\
\text { programmé }\end{array}$ & $\begin{array}{l}\text { Antibiothérapie par IV tous les } 3 \text { mois. } \\
\text { Alternative : aérosolthérapie par tobramycine } 300 \mathrm{mg} 2 \text { fois par jour } \\
\text { durant } 28 \text { jours puis } 28 \text { jours sans traitement (à partir de } 6 \text { ans, et durée } \\
\text { maximum validée par l'AMM de } 96 \text { semaines). } \\
\text { Si apparition de signes d'aggravation clinique ou fonctionnelle respi- } \\
\text { ratoire, retour à la voie IV. }\end{array}$ \\
\hline
\end{tabular}

AMM : autorisation de mise sur le marché ; IV : intraveineuse

Tableau II. Résistances acquises, phénotypes de PA. D'après ${ }^{[6]}$ (avec l'aimable autorisation des éditions Biomérieux et des auteurs).

\begin{tabular}{lcccccc}
\hline & Sauvage & Case & Gyrase & G/Nt & T/Nt/A & G/T \\
\hline Ceftazidime & $\mathrm{S}$ & $\mathrm{I} / \mathrm{R}$ & & & & \\
Ciprofloxacine & $\mathrm{S}$ & $\mathrm{S}$ & $\mathrm{R}$ & & & \\
Gentamicine & $\mathrm{S}$ & $\mathrm{S}$ & & $\mathrm{R}$ & $\mathrm{S}$ & $\mathrm{R}$ \\
Tobramycine & $\mathrm{S}$ & $\mathrm{S}$ & & $\mathrm{S}$ & $\mathrm{R}$ & $\mathrm{R}$ \\
Amikacine & $\mathrm{S}$ & $\mathrm{S}$ & & $\mathrm{S}$ & $\mathrm{R}$ & $\mathrm{S}$ \\
Colistine & $\mathrm{S}$ & $\mathrm{S}$ & $\mathrm{S}$ & $\mathrm{S}$ & $\mathrm{S}$ & $\mathrm{S}$ \\
\hline
\end{tabular}

$\mathbf{A}$ : amikacine ; Case : céphalosporinase ; $\mathbf{G}$ : gentamicine $; \mathbf{I}:$ intermédiaire $; \mathbf{N t}:$ nétromicine $; \mathbf{R}:$ résistant $; \mathbf{S}:$ sauvage ; $\mathbf{T}:$ tobramycine

de l'infection chronique, la bonne tolérance, l'efficacité clinique prouvée et l'absence de coût supplémentaire, font de l'antibiothérapie inhalée un atout thérapeutique dans la prise en charge des infections respiratoires à $P A$.

\section{Bactériologie}

$P A$ est un bacille à Gram négatif notamment impliqué dans les surinfections pulmonaires chez les patients atteints de mucoviscidose. Il est ubiquitaire, saprophyte, et se développe dans un environnement humide sous forme de microcolonies. $P A$ a toujours été considéré comme une cible difficile en chimiothérapie anti-infectieuse. ${ }^{[5]}$ En effet, la résistance aux antibiotiques est souvent native, mais peut-être aussi acquise. Ces résistances sont parfois associées entre elles, transitoires et adaptatives, c'est-à-dire favorisées par la pression de sélection de l'antibiothérapie. Le mécanisme de résistance acquise diffère selon le phénotype de $P A$ (tableau II). ${ }^{[6]}$

Du fait de ces résistances associées, l'incidence des souches de PA multirésistantes est en constante augmentation, rendant la détermination de la sensibilité des isolats essentielle. ${ }^{[7]} P A$ est naturellement résistant aux aminopénicillines, aux céphalosporines de première et deuxième génération et aux quinolones classiques.
La sensibilité de $P A$ est plus faible pour la pipéracilline $(80 \%)$ que pour l'imipénème $(84 \%)$ et la ceftazidime $(86 \%)$ et la sensibilité pour la ciprofloxacine (environ $69 \%$ ) est moins fréquente que pour les bêta-lactames (tableau III). ${ }^{[8]}$

Dans une étude menée sur des patients atteints de mucoviscidose, et visant à déterminer la sensibilité de $P A$ aux antibiotiques couramment utilisés chez cette population, ceux-ci ont été classés par ordre de résistance décroissante. Le taux de résistance de 1240 isolats (508 patients) a été le plus faible pour la tobramycine $(5,4 \%)$, puis la ceftazidime $(11,1 \%)$, l'aztréonam $(11,9 \%), 1$ 'amikacine $(13,1 \%)$, la ticarcilline $(16,7 \%)$, la gentamicine $(19,3 \%)$ et enfin la ciprofloxacine $(20,7 \%)$. ${ }^{[9]}$

Une infection par des souches résistantes ne doit pas être négligée, car elle multiplie par trois la mortalité, par neuf le risque de bactériémie secondaire, et par deux la durée d'hospitalisation et le coût de prise en charge du patient. ${ }^{[10]}$

\section{L'aérosolthérapie}

L'administration d'antibiotiques par voie inhalée est une pratique ancienne, initiée en 1944 de façon empirique au cours des infections respiratoires de la mucoviscidose. Depuis une décennie, elle fait l'objet d'une évaluation scientifique réelle. Cependant, 
Tableau III. Pseudomonas aeruginosa : sensibilité aux antibiotiques. D’après ${ }^{[8]}$

\begin{tabular}{|c|c|c|c|c|}
\hline \multicolumn{2}{|c|}{ Antibiotiques } & \multicolumn{3}{|c|}{$\%$ de souches } \\
\hline & & Sensible & Intermédiaire & Résistant \\
\hline \multirow{4}{*}{$\beta$ - lactamines } & Ticarcilline & 60,6 & 6,5 & 33,0 \\
\hline & Pipéracilline & 80,3 & 5,2 & 14,6 \\
\hline & Ceftazidime & 85,6 & 8,2 & 6,2 \\
\hline & Imipénème & 83,8 & 4,1 & 12,1 \\
\hline \multirow{3}{*}{ Aminosides } & Amikacine & 83,0 & 6,8 & 10,2 \\
\hline & Gentamicine & 70,0 & 8,8 & 21,2 \\
\hline & Tobramycine & 82,8 & 1,3 & 15,9 \\
\hline Fluoroquinolones & & 68,9 & 3,9 & 27,2 \\
\hline
\end{tabular}

même les études les plus récentes concluent à l'heure actuelle à l'impossibilité de choisir parmi l'arsenal thérapeutique l'antibiotique inhalé idéal pour traiter ces infections. ${ }^{[11]}$

L'antibiothérapie par voie inhalée est néanmoins devenue une approche précieuse, pour le traitement des patients infectés par $P A$ et atteints de mucoviscidose, car elle cible directement le site de l'infection. Comparativement à une dose administrée par voie systémique, le traitement aérosol permet de plus fortes concentrations d'antibiotiques au niveau pulmonaire et réduit le niveau d'exposition systémique au médicament et donc sa toxicité. ${ }^{[12]}$ Enfin, l'impact budgétaire a également été appréhendé dans une étude sur 4 ans. Une augmentation de $20 \%$ de l'utilisation de solution inhalée de tobramycine a évidemment entraîné un surcoût direct, mais a surtout eu pour conséquence une réduction globale des coûts par le biais de la diminution du recours aux antibiotiques injectables $(-4 \%)$ et de la diminution des hospitalisations $(-3 \%){ }^{[13]}$

Cependant, les modifications pulmonaires inhérentes à la mucoviscidose et à l'infection par $P A$ peuvent diminuer notablement l'efficacité de l'aérosolthérapie par rapport aux patients non atteints de mucoviscidose. Il convient donc d'évaluer soigneusement chaque paramètre de l'aérosolthérapie, en les adaptant aux patients atteints de mucoviscidose. Le choix de l'appareil de nébulisation doit également se faire en fonction des caractéristiques propres de chaque produit. Ainsi, deux appareils de nébulisation (eFlow rapid et LC plus), ayant une efficacité similaire chez les patients sains donnent des efficacités différentes chez les patients atteints de mucoviscidose. ${ }^{[14]}$ De plus, lorsque des études pharmacocinétiques ont été conduites avec un matériel donné, le clinicien utilisant un autre appareil de nébulisation peut être amené à modifier les doses recommandées, pour obtenir une efficacité équivalente. ${ }^{[15]}$

\subsection{Aminosides}

\subsubsection{Tobramycine}

Les aminosides possèdent plusieurs avantages en tant que traitement anti- $P A$ par aérosolthérapie : leurs effets post-antibiotiques sont longs, et ils ont un goût acceptable. Parmi eux, la tobramycine possède une des toxicités systémiques les plus faibles, la voie inhalée permettant, en outre, d'obtenir un dépôt pulmonaire important associé à de très faibles concentrations plasmatiques. ${ }^{[16]}$ Plusieurs études ont évalué l'innocuité de la tobramycine par voie inhalée, et aucune oto- ou nephrotoxicité n'ont été retrouvées chez 22 patients durant 12 semaines $^{[17]}$ et chez 14 patients durant 20 mois. ${ }^{\text {[18] }}$

En France, une solution de tobramycine pour inhalation $\left(\right.$ Tobi $\left.^{\circledR}\right)$, a obtenu l'autorisation de mise sur le marché (AMM) en 2000 pour les sujets de plus de 6 ans atteints de mucoviscidose, avec une posologie préconisée de $300 \mathrm{mg}$ deux fois par jour, quel que soit l'âge. ${ }^{[19]}$

\subsubsection{Primocolonisation à Pseudomonas aeruginosa}

Dans le cadre de l'éradication de $P A$ lors d'infections précoces, la tobramycine en solution inhalée a montré son efficacité lors d'une étude randomisée en double aveugle versus placebo. ${ }^{\text {[20] }}$ Chez 8 enfants de moins de 6 ans, $300 \mathrm{mg}$ de tobramycine inhalée deux fois par jour durant 28 jours a réduit le nombre de colonyforming unit (CFU) de $P A$ de $5,25 \log _{10} / \mathrm{mL}$ (versus une augmentation de $0,30 \log _{10} / \mathrm{mL}$ pour le placebo, $p<0,0001$ ).

L'essai early inhaled tobramycin for eradication (ELITE) a évalué l'efficacité et la tolérance d'une solution pour inhalation de tobramycine, à la posologie de $300 \mathrm{mg}$ deux fois par jour pendant 28 jours $(n=45)$ ou pendant 56 jours $(n=43)$, dans le traitement d'une primocolonisation à $P A$ chez des patients de 6 ans ou plus, atteints de mucoviscidose. Cette étude a mis en évidence une efficacité et une tolérance similaires pour des durées de traitement de 28 et 56 jours (respectivement, éradication de $P A$ pour $93 \%$ et $92 \%$ des patients 1 mois après traitement et date de recolonisation par $P A$ non statistiquement significative). ${ }^{[21]}$

L'éradication de $P A$ pouvant donc être obtenue après 28 jours de traitement (300 mg deux fois par jour), une autre étude a évalué la prolongation des effets du traitement. ${ }^{[22]}$ Les 31 patients (âge entre 6 mois et 6 ans) ont été divisés en plusieurs cohortes (durée du traitement/jour de contrôle post traitement). L'éradication de 
$P A$ a été confirmée pour $75 \%$ des patients cohorte 28/56, $63 \%$ pour la cohorte $28 / 84,82 \%$ pour la cohorte $56 / 112$ et $75 \%$ pour la cohorte $28 / 112$. Cette étude met en évidence une persistance des effets anti- $P A$ du traitement par tobramycine inhalée jusqu'à 3 mois après traitement. Les auteurs modèrent ces affirmations en distinguant les effets significatifs observés au niveau de la partie basse du tractus respiratoire, alors que les effets sur la partie haute (oropharyngée) semblent plus limités.

Une autre étude a envisagé l'administration de plus faibles doses de tobramycine en continu durant 12 mois ( $80 \mathrm{mg}$ deux fois par jour). ${ }^{[23]}$ Vingt-deux patients d'âge supérieur à 4 ans ont été randomisés dans les groupes tobramycine ou placebo. Ce mode d'administration a montré son efficacité et son innocuité par rapport au placebo, et le temps moyen de disparition des CFU de $P A$ a été de 1,89 mois. Durant cette étude, $P A$ a disparu des prélèvements pour 8 patients sur 9 du groupe tobramycine et aucun nouvel épisode infectieux à $P A$ n'a été constaté pour la durée de l'étude. Deux patients ont arrêté l'étude dans le groupe tobramycine et 6 dans le groupe placebo, ce qui rend ces résultats difficilement interprétables. Un autre travail ayant inclus 15 patients (âge entre 1 et 22 ans) vient cependant confirmer ces résultats (prélèvements exempts de $P A$ pour 14 patients sur 15 du groupe tobramycine durant un an), en montrant également l'absence d'émergence de souches de $P A$ résistantes à la tobramycine durant la période de l'étude. ${ }^{[24]}$

\subsubsection{Infections chroniques à Pseudomonas aeruginosa}

L'efficacité et l'innocuité de la tobramycine en solution inhalée ont également été évaluées dans le traitement de l'infection chronique à $P A$ chez le patient atteint de mucoviscidose.

L'efficacité de la tobramycine en solution inhalée, à une dose de $300 \mathrm{mg}$ deux fois par jour a été évaluée versus placebo pour une cohorte de 520 patients d'âge supérieur à 6 ans. Le protocole se déroulait sur une durée totale de 24 semaines, par cycles de 28 jours de traitement puis 28 jours sans traitement. La tobramycine a montré une efficacité significative sur les paramètres infectieux (réduction de $0,8 \log _{10} \mathrm{CFU}$ de $P A / \mathrm{mL}$, versus augmentation de 0,3 pour le placebo, $p<0,001$ ) ainsi que sur les paramètres respiratoires (amélioration de $10 \%$ du volume expiratoire maximum par seconde [VEMS]) et de $8 \%$ de la capacité vitale forcée $[\mathrm{CVF}])$, versus diminution respective de $2 \%$ et $1 \%$ pour le placebo, $p<0,001)$. Cette étude met également en évidence des effets maximum sur les paramètres respiratoires pour la sous-population d'âges compris entre 13 et 17 ans ( $\mathrm{n}=120$, amélioration du VEMS de 15,9\%, versus diminution de 7,1\% pour le groupe placebo, $p<0,001) .{ }^{[25]}$ Dans une étude à plus court terme de 4 semaines, versus placebo, l'administration de tobramycine solution inhalée à $600 \mathrm{mg}$ trois fois par jour a permis une réduction de $2,1 \log _{10} \mathrm{CFU}$ de $P A / \mathrm{mL}$ versus réduction de 0,2 pour le bras placebo. Les paramètres respiratoires ont également été significativement améliorés (amélioration de 3,7\% du VEMS et de $2,1 \%$ de la CVF, versus réduction respectivement de $6 \%$ et $4,1 \%$ pour le placebo). ${ }^{[26]}$

Un essai clinique randomisé a également comparé l'efficacité de la tobramycine (300 mg deux fois par jour) et de la colistine (Colimycine ${ }^{\circledR}, 80 \mathrm{mg}$ deux fois par jour) en aérosolthérapie pour une cohorte de 115 patients âgés de 6 ans et plus ayant un VEMS supérieur ou égal à $25 \%$. ${ }^{[27]}$ Cet essai a permis de conclure à un avantage léger en faveur de la tobramycine pour les paramètres infectieux (réduction de $0,9 \log _{10} \mathrm{CFU}$ de $P A / \mathrm{mL}$ versus 0,6 ), et un avantage significatif pour les paramètres respiratoires (amélioration de 6,7 $\pm 15,1 \%$ du VEMS versus amélioration de $0,4 \pm 18,8 \%, p=0,008)$. De plus, une analyse des sous-populations par âge a été effectuée bien que comportant un faible effectif (entre 11 et 34 patients par sous-groupes) rendant les résultats peu significatifs. Cette analyse suggère que la tobramycine en solution inhalée est nettement supérieure à la colistine, au niveau des paramètres respiratoires chez le patient d'âge inférieur à 6 ans (amélioration de 11,5 \pm 24,3\% du VEMS versus diminution de $8,1 \pm 18,4 \%$ ), alors que cet avantage se réduit considérablement chez le patient d'âge supérieur à 18 ans. Cependant, cette étude n'a pas été réalisée en double aveugle, environ $80 \%$ de la cohorte ayant été traité auparavant par de la colistine. Par ailleurs l'administration des deux principes actifs n'a pas été effectuée avec le même modèle de nébulisateur. Une étude en double aveugle tobramycine versus colistine apparaît donc nécessaire pour confirmer ces résultats.

\subsubsection{Impacts secondaires de l'aérosolthérapie par tobramycine}

L'utilisation prolongée de tobramycine (28 jours de traitement suivis de 28 jours sans traitement, répétés 3 fois) ne semble pas augmenter l'émergence de souches bactériennes naturellement résistantes à la tobramycine. ${ }^{[28]}$ Un traitement intermittent semble même, par rapport au placebo, diminuer l'émergence de bactéries de type Stenotrophomonas maltophilia et Alcaligenes xylosoxidans. Cependant, il favorise l'augmentation de l'émergence de Candida albicans (22\% versus $16 \%$ pour le placebo, $p=0,06)$ et d'Aspergillus sp. (18\% versus $8 \%, p=0,001)$, mais celle-ci est sans incidence clinique dans la cohorte étudiée ( $\mathrm{n}=520)$. Cette même étude montre également l'augmentation significative d'isolats de $P A$ résistants, ayant des concentrations minimales inhibitrices (CMI) supérieures à $16 \mu \mathrm{g} / \mathrm{mL}$ (semaine 24, $23 \%$ dans le bras tobramycine versus $8 \%$ dans le bras placebo, $p<0,001$ ), mais insiste sur la faible répercussion clinique de cette augmentation et précise que le traitement intermittent aux posologies usuelles diminue la pression de sélection favorisant cette émergence. Il a d'ailleurs été montré que l'utilisation de la 
tobramycine en solution inhalée de manière intermittente permet d'obtenir un pourcentage de $95,1 \%$ des isolats de $P A$ sensibles à la tobramycine. ${ }^{[29]} \mathrm{Il}$ est également indiqué que d'autres protocoles (tobramycine $600 \mathrm{mg}$, trois fois par jour, traitement continu de 3 mois) entrainent des baisses de sensibilité de $P A$ plus conséquentes. ${ }^{[28]}$ A contrario, les augmentations d'isolats de $P A$ multirésistants apparaissent identiques dans les deux cohortes.

\subsubsection{Nouvelles formes galéniques}

De nouvelles formes galéniques sont également à l'étude afin d'améliorer la distribution pulmonaire de la tobramycine. Ainsi, l'essai EVOLVE a étudié l'efficacité et la tolérance de la tobramycine en poudre pour inhalation versus placebo. ${ }^{[30]}$ Les résultats de cet essai montrent que cette forme galénique permet un temps d'administration réduit (4 à 6 min) par rapport à celui de la solution de tobramycine pour inhalation (15 min), pour une efficacité comparable. Cette réduction significative permettrait une meilleure observance du patient atteint de mucoviscidose.

Récemment, l'essai EAGER a également comparé l'efficacité et la tolérance de la tobramycine en poudre versus en solution. ${ }^{[31]}$ Cinq cent trente-trois patients ont été randomisés dans deux groupes : TIP (112 mg de tobramycine en poudre inhalée) et TIS (300 mg de tobramycine en solution inhalée). Le protocole d'administration était identique (deux fois par jour durant 28 jours, 3 cycles), mais l'appareil de nébulisation différait pour les deux groupes. L'étude s'appuie sur l'intérêt d'un temps d'administration plus court pour le groupe TIP (5,6 min versus 19,7 min). Cependant, il n'apparaît aucune différence significative sur les améliorations de la fonction pulmonaire (VEMS) ou la densité bactérienne. La tolérance dans le groupe TIP a été significativement inférieure que dans le groupe TIS (effets indésirables $90,3 \%$ versus $84,2 \%, p=0,05$ ), avec notamment une incidence de toux significativement plus élevée dans le groupe TIP $(48,4 \%$ versus $31,1 \%$ ), ainsi que l'apparition de dysphonies (13,6\% versus $3,8 \%$ ) et de dysgueusies (3,9\% versus $0,5 \%$ ). L'incidence des arrêts de traitements a été supérieure pour le groupe TIP (26,9\% versus 18,2\%), mais l'indice de satisfaction globale des patients du groupe TIP a été significativement meilleur (76,2\% versus $71,0 \%, p=0,0018)$.

\subsubsection{Amikacine sous forme liposomale}

Une forme liposomale d'amikacine pour inhalation a été évaluée dans un modèle in vitro. ${ }^{[32]}$ les chercheurs ont montré l'avantage des liposomes du fait de leur pénétration dans le biofilm de $P A$. Des études in vivo sont nécessaires, mais le principe d'introduire l'antibiotique directement dans le biofilm est séduisant. Les liposomes libèrent le médicament avec une cinétique qui prolonge le temps de séjour dans les voies respiratoires et peut réduire la fréquence d'administration à une fois par jour. Une étude pharmacocinétique et pharmacodynamique a été menée dans une cohorte de 24 patients atteints de mucoviscidose et chroniquement infectés par $P A$ (âge $\geqslant 13$ ans, et VEMS $\geqslant 40 \%$ ). Les patients recevaient $500 \mathrm{mg}$ d'amikacine en $40 \mathrm{~min}$ d'inhalation. Une amélioration significative du VEMS a été observée après 7 jours (amélioration de $10,8 \%, p<0,001)$ mais semble s'atténuer à 14 jours de traitement (amélioration de 5,6\%,p=0,073). ${ }^{[33]}$

Les résultats des études de phase II versus placebo ont été récemment communiqués lors d'un congrès. ${ }^{[34,35]}$ Une cohorte de 105 patients atteints de mucoviscidose chroniquement infectés par $P A$ (âge $\geqslant 6$ ans, et VEMS $\geqslant 40 \%$, patients non traités par antibiothérapie inhalée depuis au moins 28 jours) a été randomisée soit dans le groupe placebo, soit dans le groupe amikacine liposomale pour inhalation (amikacine liposomale, Arikace ${ }^{\circledR}$ ). Quatre doses (70 mg, $140 \mathrm{mg}, 280 \mathrm{mg}$ et $560 \mathrm{mg}$ ) ont été testées, en une administration par jour durant 28 jours. La tolérance de l'amikacine liposomale s'est avérée comparable au placebo. Les améliorations des paramètres respiratoires ont été significatifs aux doses de $280 \mathrm{mg}$ et $560 \mathrm{mg}$, avec un effet prolongé un mois après traitement pour la posologie de $560 \mathrm{mg}$ (amélioration de 12,5\% du VEMS, $p=0,003$ ). De même, la posologie de $560 \mathrm{mg}$ durant un mois a permis une réduction significative des isolats de $P A$ $\left(-1,7 \log _{10} \mathrm{CFU}, p=0,007\right)$. Enfin, le temps de survenue d'exacerbations pulmonaires a été également rallongé (45,3 jours versus 31,4 pour le placebo). L'Arikace ${ }^{\circledR}$ était en étude de phase III mais la Food and drug administration (FDA) a récemment informé de l'interruption de l'essai suite aux résultats préliminaires de carcinogénicité à long terme chez l'animal (rat). Cet essai reste suspendu tant que la FDA n' aura pas notamment reçu des résultats de carcinogénicité à long terme effectués sur un autre modèle animal (chien), afin de confirmer ou d'infirmer les résultats obtenus chez le rat. ${ }^{[36]}$

\subsection{Colistine}

La colistine est un antibiotique de la famille des polymyxines, dont le mode d'action consiste en une déstabilisation de la structure de la membrane externe des bactéries. Son spectre d'action est relativement étroit (certains bacilles gram négatif), mais sa bonne activité sur $P A$ en fait un bon candidat dans l'arsenal thérapeutique destiné au patient atteint de mucoviscidose. Cependant, sa toxicité non négligeable, néphrotoxicité et neurotoxicité notamment, oriente plutôt vers un usage par aérosolthérapie bien que des études récentes suggèrent que ces toxicités ont été surévaluées. ${ }^{[37,38]}$

La colistine existe sous deux formes commerciales : le sulfate de colistine et le méthanesulfonate de colistine (sel de sodium). 
Seule la forme méthanesulfonate devrait être utilisée sous forme nébulisée dans le traitement des infections à $P A$. En effet, une étude a comparé la tolérance des deux formes administrées par nébulisation à des patients atteints de mucoviscidose. La forme sulfate de colistine a entrainé une diminution significative de la fonction pulmonaire (30 min après aérosolthérapie, diminution de $10,2 \%$ du VEMS versus $2,9 \%$ pour la forme méthanesulfonate et diminution de $10,5 \%$ de la CVF versus $2,1 \%, p=0,034$ et $0,01)^{[39]}$

Le méthanesulfonate de colistine $\left(\right.$ Colistine $\left.^{\circledR}\right)$ a ainsi obtenu l'AMM, notamment dans l'indication du traitement des infections à $P A$ chez le patient atteint de mucoviscidose. La posologie recommandée est de 1 à 6 millions d'unités (MU) par jour en une à trois séances d'inhalation (soit $80 \mathrm{mg}$ à $480 \mathrm{mg}$ ). La reconstitution de la solution pour inhalation doit toujours s'effectuer extemporanément. Un cas clinique présente, en effet, un cas de syndrome de détresse respiratoire aiguë fatal chez une patiente de 29 ans atteinte de mucoviscidose, probablement attribué à une solution de colistine reconstituée depuis 5 semaines. ${ }^{[40]}$

Les propriétés pharmacocinétiques de la colistine ont été étudiées. ${ }^{[41]}$ Les concentrations plasmatiques atteignent leur maximum 1,5 h après administration et, en moyenne, 4,3 $\pm 1,3 \%$ de la dose inhalée est détectée dans les urines. Les concentrations maximales dans les expectorations (pic $1 \mathrm{~h}$ après aérosolthérapie) sont au moins 10 fois plus élevées que la CMI de $P A$. Ces concentrations restent encore aux environs de $4 \mathrm{mg} / \mathrm{L} 12$ heures après traitement.

\subsubsection{Primocolonisation à Pseudomonas aeruginosa}

L'efficacité de l'aérosolthérapie de colistine n'a pas été démontrée en traitement prophylactique de l'infection à $P A$ chez le patient atteint de mucoviscidose. ${ }^{[42]}$

En revanche, plusieurs études se sont intéressées à l'utilisation conjointe de la colistine en aérosolthérapie et de la ciprofloxacine per os dans le traitement de la primocolonisation à $P A$. Il a ainsi été montré, par rapport au placebo, une réduction de $58 \%$ à $14 \%$ du passage à l'infection chronique, lors de l'utilisation de colistine $1 \mathrm{MU}$ deux fois par jours associée à la ciprofloxacine. ${ }^{[43]}$ De même, l'association ciprofloxacine per os $30-40 \mathrm{mg} / \mathrm{kg} / \mathrm{j}$ et colistine inhalée $1 \mathrm{MU}$ deux fois par jour en traitement d'entretien durant deux semaines, a permis de réduire la prévalence de $P A$ de $86 \%$ (groupe contrôle) à 4,6\% sous traitement. ${ }^{[4]}$ Cependant, le faible effectif de la cohorte étudiée (16 patients) vient tempérer ces résultats. Une étude sur une cohorte de 146 patients suivis durant 15 ans rapporte également que l'instauration systématique de colistine inhalée associée à de la ciprofloxacine per os, dès que $P A$ est isolé, permet de prévenir le passage à l'infection chronique pour $80 \%$ des patients atteints de mucoviscidose. ${ }^{[45]}$ Une autre étude (associations de ciprofloxacine per os $30 \mathrm{mg} / \mathrm{kg} / \mathrm{j}$ deux fois par jour et de colistine $1 \mathrm{MU}$ deux fois par jour ou $2 \mathrm{MU}$ trois fois par jour jusqu'à 3 mois si $P A$ persiste) a permis également d'observer l'éradication de $P A$ pour $90 \%$ des patients $(n=20) .{ }^{[46]}$

Aucune différence significative d'efficacité n'a été retrouvée dans une cohorte de 81 patients atteints de mucoviscidose entre l'utilisation de colistine $30 \mathrm{mg}$ ou $75 \mathrm{mg}$ deux fois par jour. ${ }^{[47]}$

\subsubsection{Infections chroniques à Pseudomonas aeruginosa}

A contrario, les études ciblant l'utilisation de la colistine dans les infections chroniques à $P A$ sont peu nombreuses. L'étude de référence est une étude randomisée en double aveugle versus placebo. Quarante patients ont reçu soit $1 \mathrm{MU}$ deux fois par jour de colistine, soit un placebo. Après trois mois de traitement, il a été noté pour le bras colistine une diminution de $7 \%$ de la capacité vitale $(\mathrm{CV})$ et une diminution de $11 \%$ du VEMS versus des diminutions respectives de $18 \%$ et $17 \%$ pour le groupe placebo. ${ }^{[48]}$

\subsubsection{Impacts secondaires de l'aérosolthérapie par colistine}

L'émergence de souches de $P A$ résistantes respectivement à la tobramycine ou à la colistine chez des patients atteints de mucoviscidose ayant une infection chronique a été comparée. Sur la cohorte de 22 patients étudiée (1 844 isolats de $P A$ ), une résistance à la tobramycine a été retrouvée dans $27,5 \%$ des isolats, alors que tous les isolats sont restés sensibles à la colistine. ${ }^{[49]}$ Cette étude montre que $P A$ présente un faible taux de résistance à la colistine. L'utilisation de la colistine est ainsi recommandée dans le cadre du traitement de souches de $P A$ multirésistantes. ${ }^{[50]} \mathrm{Ce}-$ pendant, une étude récente tempère ces affirmations en mettant en évidence un taux non négligeable de fausses sensibilités aux polymyxines selon les méthodes employées (taux d'erreur négligeable pour les dilutions dans la gélose, de $12 \%$ pour les microdilutions de bouillon et $18 \%$ pour l'Etest et la diffusion de disques). ${ }^{[51]}$

Ce faible taux d'émergence de souches de $P A$ résistantes est cependant un avantage majeur de la colistine par aérosolthérapie. Ainsi, une étude s'est intéressée à l'utilisation de celle-ci dans la prise en charge de patients atteints de mucoviscidose en attente de greffe pulmonaire et infectés par $P A$ multi-résistant. L'initiation de l'immunosuppression et la multi-résistance limitant considérablement l'arsenal thérapeutique, les antibiotiques injectables ont été stoppés, et 20 patients placés sous aérosolthérapie de colistine $75 \mathrm{mg}$ deux fois par jour. Le temps d'apparition de souches sensibles à la colistine a été de 45,1 $\pm 20,2$ jours (versus $144,6 \pm 48,0$ jours pour les patients non traités par colistine). ${ }^{[52]}$ 


\subsection{Monobactam : lysinate d'aztréonam}

Le lysinate d'aztréonam, un monobactame salifié, est une nouvelle forme galénique d'antibiotique inhalée utilisée dans le traitement des infections à $P A$ chez les patients atteints de mucoviscidose. La forme standard par voie intraveineuse de l'aztréonam contient de l'arginine qui s'est révélée inflammatoire lors d'inhalation chronique. ${ }^{[53,54]}$ Par conséquent, le médicament a été reformulé en sel de lysine pour inhalation. Cette forme galénique pour voie inhalée se distingue par un temps d'administration relativement court (moins de $3 \mathrm{~min}$ ).

Une étude de pharmacocinétique et de tolérance a comparé des doses de 75, 150 et $225 \mathrm{mg}$ ainsi qu'un placebo. Toutes les doses ont été tolérées par 23 patients sur 24, le dernier ayant eu une diminution asymptomatique du VEMS à partir de $150 \mathrm{mg}$. Les concentrations en aztréonam dans les expectorations ont toutes été supérieures ou égales au $\mathrm{CMI}_{50}$ durant au moins $4 \mathrm{~h}$ après traitement. À la dose de $75 \mathrm{mg}$, les paramètres pharmacocinétiques ont été calculés : concentration plasmatique maximale $\left(\mathrm{C}_{\max }\right)=$ $419 \mathrm{ng} / \mathrm{g}$, temps auquel est obtenue la concentration plasmatique maximale $\left(\mathrm{T}_{\max }\right)=0,99 \mathrm{~h}, \mathrm{t}_{1 / 2}=2,1 \mathrm{~h} .{ }^{[55]}$

Récemment, l'aztréonam en solution pour inhalation $\left(\right.$ Cayston $\left.{ }^{\circledR}\right)$ a obtenu une AMM Européenne comme traitement améliorant les symptômes respiratoires chez le patient atteint de mucoviscidose. La posologie recommandée est de $75 \mathrm{mg}$ trois fois par jour durant 28 jours.

Une étude de phase II sur une cohorte de 105 patients atteints de mucoviscidose chroniquement infectés par $P A$ a comparé l'utilisation d'aztréonam $75 \mathrm{mg}$ deux fois par jour versus $225 \mathrm{mg}$ deux fois par jour et versus placebo durant 14 jours. La réduction de la densité de CFU de $P A$ a été significativement plus importante pour ces deux groupes versus placebo $(p<0,001)$. Le traitement par aztréonam n'apporte globalement aucune amélioration significative du VEMS à J14, bien que cette amélioration puisse exister dans certains sous-groupes et que l'utilisation de bronchodilatateurs semble nettement augmenter l'efficacité de l'aztréonam. Aucune différence significative de tolérance n'a été notée entre les 3 bras, malgré une tendance à l'augmentation de symptômes respiratoires dans le bras $225 \mathrm{mg}$. Ces résultats médiocres peuvent être attribués à la faible durée de traitement (14 jours) et aux bons paramètres pulmonaires initiaux de la cohorte étudiée (critère d'inclusion : VEMS $\geqslant 40 \%$, VEMS moyen $=77,3 \%$ ). ${ }^{[56]}$

L'administration de $75 \mathrm{mg}$ d'aztréonam 3 fois par jour chez le patient atteint de mucoviscidose chroniquement infecté par $P A$ a été étudiée versus placebo dans deux essais de phase III. L'essai AIR-CF1 comprenait une cohorte de 164 patients ayant un VEMS compris entre $25 \%$ et $75 \%$ n'ayant pas eu d'antibiothérapie récente, et l'essai AIR-CF2 une cohorte de 211 patients habituellement traités par des solutions inhalées de tobra- mycine. Après 28 jours de traitement, les scores de qualité de vie et de fonction pulmonaire ont été significativement améliorés $(\mathrm{VEMS}+10,3 \%$ pour AIR-CF1 et $+6,3 \%$ pour AIR-CF2, $p \leqslant$ 0,001). De même, le temps nécessaire au recours de traitements lors d'exacerbations pulmonaires a été allongé (92 jours versus $71, p=0,007)$, et le nombre de $P A$ dans les expectorations a diminué $\left(-1,453 \log _{10} \mathrm{CFU} / \mathrm{g}\right.$ pour AIR-CF1 et $-0,66 \log _{10} \mathrm{CFU} / \mathrm{g}$ pour AIR-CF2 $p<0,001$ et $p=0,007)$. Aucun problème de tolérance n'a été noté, et la sensibilité de $P A$ à l'aztréonam était identique au début et à la fin de l'étude. ${ }^{[57,58]}$

Ces résultats ont été confirmés durant 18 mois d'utilisation cyclique (essai AIR-CF3), et ont mis en évidence un taux important d'observance de la part des patients (92\% pour le groupe « deux administrations par jour» et $88 \%$ pour «trois administrations par jour »). Cette étude montre également un avantage en faveur de l'administration trois fois par jour, en termes de fonction pulmonaire et de symptômes respiratoires. ${ }^{[59]}$

Récemment, le laboratoire commercialisant l'aztréonam en solution pour inhalation a affirmé que l'étude de phase III comparant l'efficacité des solutions inhalées de tobramycine et d'aztréonam (aux posologies usuelles) chez le patient atteint de mucoviscidose et infecté par $P A$ avait atteint son critère principal d'évaluation. Ainsi, l'aztréonam s'avérerait être supérieur en terme d'amélioration du VEMS après 3 cycles de traitement ( $+2,05 \%$ pour l'aztréonam versus $-0,66 \%$ pour la tobramycine). Cependant, environ $85 \%$ des patients avaient reçu au moins 3 mois de traitement par tobramycine inhalée dans les 12 mois précédant l'étude. ${ }^{[60]}$

Quelques effets secondaires ont été recensés dans les études de phase III. Il s'agit principalement d'affections respiratoires à type de toux, bronchospasme, rhinorrhée. Quelques éruptions cutanées et fièvres ont également été notées.

\subsection{Fluoroquinolones}

\subsubsection{Ciprofloxacine}

L'utilisation de la ciprofloxacine en solution par voie inhalée a été étudiée avec différents nébuliseurs. ${ }^{[61]}$ Bien que les résultats de cette étude montrent que l'utilisation d'une solution simple soit possible, les auteurs la déconseillent fortement, en l'absence d'études complémentaires.

D'autres modes de vectorisation ont également été étudiés : l'utilisation de poudres sèches de ciprofloxacine/mannitol ${ }^{[62]} \mathrm{ou}$ de ciprofloxacine/déoxyribonucléase ${ }^{[63]}$ semble prometteuse en traitant l'infection tout en favorisant la clairance du mucus.

La vectorisation de la ciprofloxacine via des liposomes est également activement étudiée. ${ }^{[64]}$ Dans une étude de phase II chez les patients atteints de bronchectasie non mucoviscidosique 
traités par ciprofloxacine sous forme liposomale, il a été noté une réduction de la charge bactérienne dans les expectorations $\left(-1,43 \log _{10} \mathrm{CFU} / \mathrm{g}\right)$ et une augmentation d'environ $7 \% \mathrm{du}$ VEMS par rapport aux valeurs initiales après 14 jours de traitement. Sur la base des caractéristiques pharmacocinétiques de cette forme galénique (demi-vie de 10,5 heures), il est possible d'obtenir un effet thérapeutique avec une administration par jour. ${ }^{[65]}$ Une étude de tolérance et d'efficacité sur une cohorte de 36 patients atteints de bronchectasie non mucoviscidosique a également comparé l'utilisation de deux doses de ciprofloxacine liposomale (150 mg et $300 \mathrm{mg}$, une fois par jour durant 28 jours). La réduction de la charge bactérienne a été respectivement de $-3,5$ et $-4 \log _{10} \mathrm{CFU} / \mathrm{g}$, avec une bonne tolérance, et l'utilisation de bronchodilatateurs n'est pas préconisée avant ou après l'aérosolthérapie. ${ }^{[66]}$ Les essais ultérieurs se focaliseront certainement sur les patients atteints de mucoviscidose, en étudiant les variations du VEMS dans cette population, mais devraient également appréhender l'émergence potentielle de souches de $P A$ résistantes.

\subsubsection{Lévofloxacine}

Le MP-376, une solution de lévofloxacine pour aérosol (Aeroquin ${ }^{\circledR}$ ) apparaît également comme une antibiothérapie anti$P A$ prometteuse chez les patients atteints de mucoviscidose.

Les tests d'efficacité réalisés sur l'animal (souris), ont montré une activité anti- $P A$ supérieure à celle de la tobramycine et de l'aztréonam utilisés par aérosolthérapie aux posologies usuelles $\left(-3,3 \log _{10} \mathrm{CFU} /\right.$ poumon versus $-2,9$ pour la tobramycine et - 1,25 pour l'aztréonam) pour un modèle d'infection chronique à $P A .{ }^{[67]} \mathrm{Il}$ apparaît dans d'autres études que la lévofloxacine serait plus efficace in vitro que la tobramycine pour éradiquer les isolats de $P A$, et que la présence de mucus ne diminuerait pas son efficacité. ${ }^{[68]}$ De plus, son efficacité ne serait également pas altérée par les conditions hypoxiques intra-pulmonaires des patients atteints de mucoviscidose. ${ }^{[69]}$ Enfin, un autre effet significatif de l'aérosolthérapie par lévofloxacine serait son action sur les cytokines pro-inflammatoires. In vitro, l'administration de doses importantes de lévofloxacine inhalée a diminué par 4 les taux d'IL-6 et par 2 les taux d'IL-8 (versus la tobramycine qui augmente les taux d'IL-6 de $50 \%$ ). Ceci permettrait un effet bénéfique sur le patient atteint de mucoviscidose indépendamment de l'effet antibactérien. ${ }^{\text {[70] }}$

Les études pharmacocinétiques et de tolérance in vivo ont montré que l'administration de lévofloxacine par aérosolthérapie était bien tolérée, et permettait d'atteindre de hautes concentrations dans les expectorations $\left(\mathrm{C}_{\max }\right.$ de 388 à $1112 \mathrm{mg} / \mathrm{L}$ en fonction de la dose versus $6 \mathrm{mg} / \mathrm{L}$ pour une administration per os). ${ }^{[71]}$

Pour tester l'efficacité et la sécurité de cette nouvelle thérapie, les chercheurs ont recruté 151 patients atteints de mucoviscidose dans une étude de phase IIb multicentrique randomisée, en double aveugle, versus placebo. Trois dosages ont été testés : $120 \mathrm{mg}$ une fois par jour, $240 \mathrm{mg}$ une fois par jour ou $240 \mathrm{mg}$ deux fois par jour. En moyenne, les patients étaient âgés de 29 ans, avaient reçu cinq antibiotiques anti pyocyaniques inhalés l'année précédant l'étude et avaient des fonctions pulmonaires généralement très altérées (VEMS $\geqslant 50 \%$ ). Après 28 jours de traitement, la quantité de $P A$ était significativement réduite dans les expectorations des patients traités par le MP-376. De plus, aucun signe de résistance n'a été mis en évidence. C'est avec le dosage le plus élevé, à savoir, $240 \mathrm{mg}$ deux fois par jour, que l'effet thérapeutique a été le plus significatif. La fonction pulmonaire s'est également améliorée chez les patients traités par le MP-376 de façon plus marquée avec la dose de $240 \mathrm{mg}$ deux fois par jour (amélioration de 10,9\% du VEMS versus placebo). En outre, le traitement par MP-376 réduit la nécessité d'un traitement avec d'autres antimicrobiens anti- $P A$ systémiques ou inhalés, avec une réduction de $79 \%$ par rapport au placebo dans le groupe traité par le MP-376 240 mg deux fois par jour. Le MP-376 a été bien toléré, sans hausse des évènements indésirables avec l'augmentation des doses, et un taux d'observance $>90 \%$ a été relevé pour plus de $80 \%$ des patients. ${ }^{[72]}$

L'objectif du MP-376 est également d'apporter aux patients une baisse significative de la charge de traitement avec deux inhalations par jour comprenant un temps de nébulisation de seulement 4 à 6 minutes chacune.

L'évaluation clinique de l'aérosol MP-376 pour le traitement des infections pulmonaires chroniques à $P A$ chez les patients atteints de mucoviscidose est en cours, une étude de phase III étant programmée à la fin de l'année. ${ }^{[73]}$

\section{Conclusion}

En France, l'espérance de vie d'un patient atteint de mucoviscidose est passée de 7 ans en 1965 à 45 ans en 2005. ${ }^{[74]}$ On peut aisément mettre en parallèle cette amélioration avec la multiplication des traitements. Le tableau IV récapitule les études visant à établir l'efficacité de ces nouvelles thérapeutiques. Il convient de garder un œil critique sur les nouvelles thérapies, l'allongement de l'espérance de vie des patients étant souvent directement relié avec un intérêt accru de l'industrie pharmaceutique pour la pathologie concernée. Parmi les antibiotiques inhalés, l'arsenal thérapeutique est actuellement en plein essor, avec de nombreuses spécialités en phase d'études cliniques (tableau V). Paradoxalement, alors que ces innovations prétendent apporter un bénéfice majeur, très peu d'études sont réalisées en comparaison avec les traitements référence (tobramycine et colistine). 
Tableau IV. Principales études sur l'aérosolthérapie antipyocyanique chez le patient atteint de mucoviscidose.

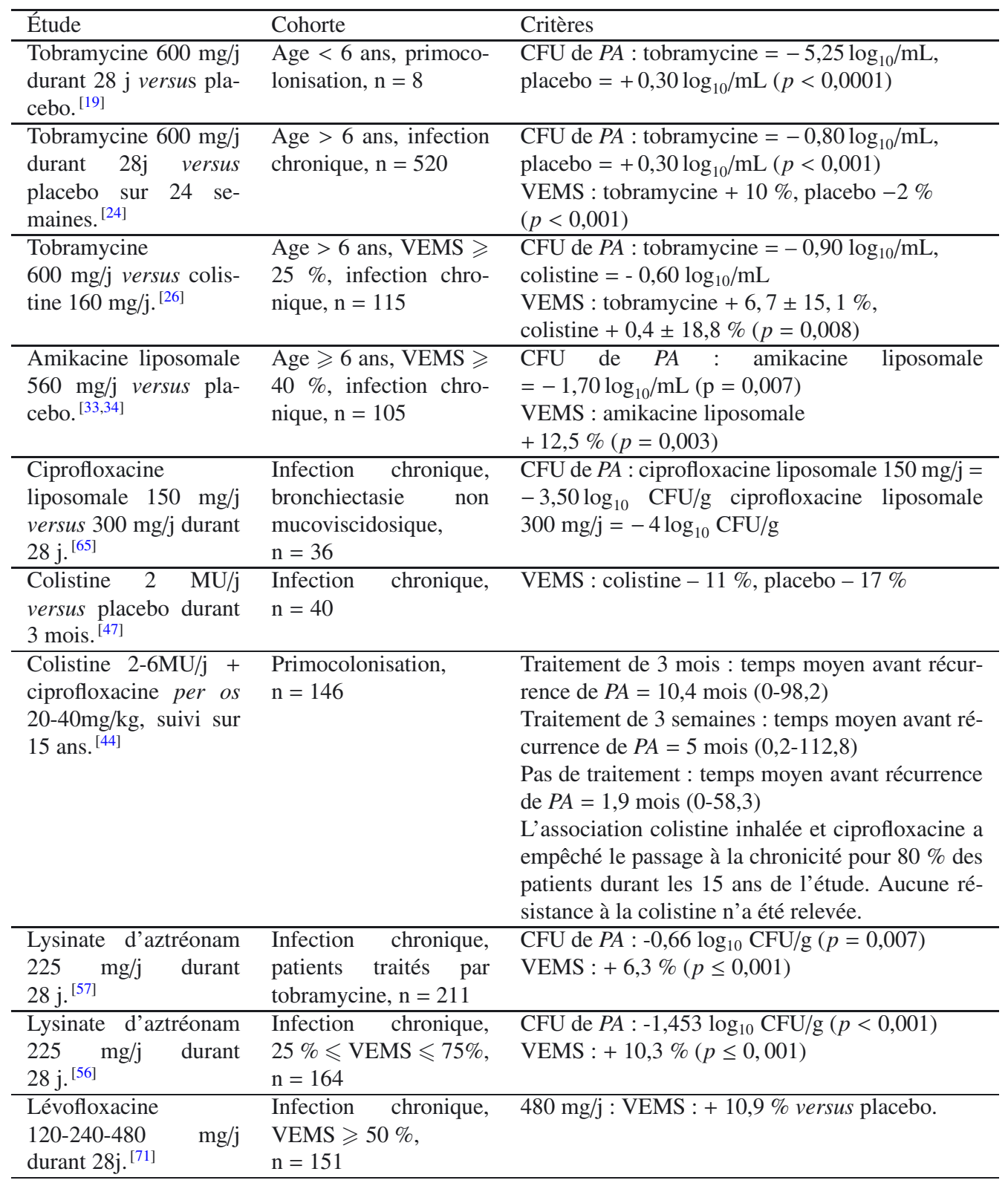

CFU : colony-forming unit; MU : millions d'unité ; PA : Pseudomonas aeruginosa; VEMS : volume expiratoire maximum par seconde

Tableau V. Essais cliniques en cours : traitement des infections à Pseudomonas aeruginosa chez les patients atteints de mucoviscidose.

\begin{tabular}{ll}
\hline \multicolumn{1}{c}{ Spécialité } & \multicolumn{1}{c}{ Études en cours } \\
\hline Tobramycine en solution & AMM, étude de phase III en cours pour enfants 3mois-6ans ${ }^{[75]}$ \\
Tobramycine en poudre & Phase III ${ }^{[76]}$ \\
Colistine & AMM \\
Lysinate d'aztréonam & AMM, étude versus tobramycine ${ }^{[60]}$ \\
Amikacine liposomale & Phase III ${ }^{[77]}$, étude versus tobramycine ${ }^{[78]}$ \\
Ciprofloxacine liposomale & Phase II (patients atteints de bronchiectasie non mucoviscidosique), ${ }^{[66]}$ Phase I/IIa $^{[79]}$ \\
Ciprofloxacine en poudre & Phase II $^{[80]}$ \\
Lévofloxacine & Phase III, $^{[73]}$ étude versus tobramycine \\
\end{tabular}

AMM : autorisation de mise sur le marché 
Dans l'attente de ces études comparatives déterminantes en termes d'amélioration du service médical rendu (ASMR), les aérosolthérapies par tobramycine et colistine devraient rester les traitements de première intention des infections à $P A$ chez le patient atteint de mucoviscidose.

Conflits d'intérêts. Les auteurs déclarent ne pas avoir de conflits d'intérêts.

\section{Références}

1. Farrell P. The prevalence of cystic fibrosis in the European Union. J Cystic Fibrosis 2008; 7: 450-3

2. Baghaie $\mathrm{N}$, Kalilzadeh $\mathrm{S}$, Hassanzad $\mathrm{M}$, et al. Determination of mortality from cystic fibrosis. Pneumologia 2010; 59:170-3

3. Emerson J, Rosenfeld M, Mc Namara S, et al. Pseudomonas aeruginosa and other predictors of mortality and morbidity in young children with cystic fibrosis. Pediatr Pulmonol 2002; 34: 91-100

4. http://www.has-sante.fr/portail/jcms/c_272206/prise-encharge-du-patient-atteint-de-mucoviscidose-pneumologieet-infectiologie

5. Barbier F, Wolff M. Multi-drug resistant Pseudomonas aeruginosa: towards a therapeutic dead end? Med Sci 2010; 26: 960-8

6. Jehl $\mathrm{F}$, Chomarat $\mathrm{M}$, Weber $\mathrm{M}$, et al. De l'antibiogramme à la prescription. Ed Biomérieux, 2000 (chap 197)

7. Tam VH, Chang KT, Abdelraouf $\mathrm{K}$, et al. Prevalence, resistance mechanisms, and susceptibility of multidrug-resistant bloodstream isolates of Pseudomonas aeruginosa. Antimicrob Agents Chemother 2010; 54: $1160-4$

8. http://www.onerba.org/IMG/pdf/onerba_rapport2007_ch6.pdf, Onerba, rapport annuel 2007

9. Shawar RM, MacLeod DL, Garber RL, et al. Activities of tobramycin and six other antibiotics against Pseudomonas aeruginosa isolates from patients with cystic fibrosis. Antimicrob Agents Chemother 1999; 43: 2877-80

10. Giamarellou H. Prescribing guidelines for severe Pseudomonas infections. J Antimicrob Chemother 2002; 49: 229-33

11. Langton Hewer SC, Smyth AR. Antibiotic strategies for eradicating Pseudomonas aeruginosa in people with cystic fibrosis. Cochrane Database Syst Rev 2009; 4: CD004197

12. Conway S. Nebulized antibiotic therapy: the evidence. Chron Respir Dis 2005; $2: 35-41$

13. Woodward TC, Brown R, Sacco P, et al. Budget impact model of tobramycin inhalation solution for treatment of Pseudomonas aeruginosa in cystic fibrosis patients. J Med Econ 2010; 13: 492-9

14. Lenney W, Edenborough F, Kho P, et al. Lung deposition of inhaled tobramycin with eFlow rapid/LC plus jet nebuliser in healthy and cystic fibrosis subjects. J Cyst Fibros 2011; 10: 9-14

15. Byrne NM, Keavey PM, Perry JD, et al. Comparison of lung deposition of colomycin using the HaloLite and the Pari LC plus nebulisers in patients with cystic fibrosis. Arch Dis Child 2003; 88: 715-8

16. Smith AL. Inhaled antibiotic therapy: What drug? What dose? What regimen? What formulation? J Cyst Fibros 2002; 1: 189-93

17. Smith A. Safety of aerosol tobramycin administration for 3 months to patients with cystic fibrosis. Pediatr Pulmonol 1989; 7: 265-71

18. Steinkamp G, Tümmler B, Gappa M, et al. Long-term tobramycin aerosol therapy in cystic fibrosis. Pediatr Pulmonol 1989; 6: 91-8

19. Sermet-Gaudelus I, Silly-Gaillez C, Claircia M, et al. Aerosols d'antibiotiques et mucoviscidose. Med Ther Ped 2000; 3: 117-24

20. Gibson RL, Emerson J, McNamara S, et al. Significant microbiological effect of inhaled tobramycin in young children with cystic fibrosis. Am J Respir Crit Care Med 2003; 167: 841-9
21. Ratjen F, Munck A, Kho P, et al. Treatment of early Pseudomonas aeruginosa infection in patients with cystic fibrosis: the ELITE trial. Thorax 2010; 65: 281-2

22. Gibson RL, Emerson J, Mayer-Hamblett N, et al. Duration of treatment effect after tobramycin solution for inhalation in young children with cystic fibrosis. Pediatr Pulmonol 2007; 42: 610-23

23. Wiesemann HG, Steinkamp G, Ratjen F, et al. Placebo-controlled, doubleblind, randomized study of aerolized tobramycin for early treatment of Pseudomonas aeruginosa colonization in cystic fibrosis. Pediatr Pulmonol 1998; 25: 88-92

24. Ratjen F, Döring G, Nikolaizik WH. Effect of inhaled tobramycin on early Pseudomonas aeruginosa colonisation in patients with cystic fibrosis. Lancet 2001; 358: 983-4

25. Ramsey BW, Pepe MS, Quan JM, et al. Intermittent administration of inhaled tobramycine in patients with cystic fibrosis. N Engl J Med 1999; 340: 23-30

26. Ramsey BW, Dorkin HL, Eisenberg JD, et al. Efficacy of aerosolized tobramycin in patients with cystic fibrosis. N Engl J Med 1993; 328: 1740-6

27. Hodson ME, Gallagher CG, Govan JRW. A randomised clinical trial of nebulised tobramycin or colistin in cystic fibrosis. Eur Respir J 2002; 20: 658-64

28. Burns JL, Van Dalfsen JM, Shawar RM, et al. Effect of chronic intermittent administration of inhaled tobramycin on respiratory microbial flora in patients with cystic fibrosis. J Infect Dis 1999; 179: 1190-6

29. Morosini MI, Garcia-Castillo M, Loza E, et al. Breakpoints for predicting Pseudomonas aeruginosa susceptibility to inhaled tobramycin in cystic fibrosis patients: use of high-range Etest strips. J Clin Microbiol 2005; 43 : 4480-5

30. Konstan MW, Geller DE, Minić P, et al. Tobramycin inhalation powder for $P$. aeruginosa infection in cystic fibrosis: The EVOLVE trial. Pediatr Pulmonol 2010; oct 20

31. Konstan MW, Flume PA, Kappler M, et al. Safety, efficacy and convenience of tobramycin inhalation powder in cystic fibrosis patients: The EAGER trial. J Cyst Fibros 2011 Jan;10(1): 54-61

32. Meers $\mathrm{P}$, Neville $\mathrm{M}$, Malinin $\mathrm{V}$, et al. Biofilm penetration, triggered release and in vivo activity of inhaled liposomal amikacin in chronic Pseudomonas aeruginosa lung infections. J Antimicrob Chemother 2008; 61: 859-68

33. Okusanya OO, Bhavnani SM, Hammel J, et al. Pharmacokinetic and pharmacodynamic evaluation of liposomal amikacin for inhalation in cystic fibrosis patients with chronic pseudomonal infection. Antimicrob Agents Chemother 2009; 53: 847-54

34. Etudes TR02-105 et TR02-106, 24 $4^{\text {th }}$ Annual North American Cystic Fibrosis Conference Poster \#243

35. Etudes TR02-105 et TR02-106, $24^{\text {th }}$ Annual North American Cystic Fibrosis Conference Poster \#227

36. http://clinicaltrials.gov/ct2/show/NCT01315691

37. Falagas ME, Kasiakou, SK. Toxicity of polymyxins: a systematic review of the evidence from old and recent studies. Crit Care 2006; 10: R27

38. Bosso JA, Liptak CA, Seilheimer DK, et al. Toxicity of colistin in cystic fibrosis patients. DICP 1991; 25: 1168-70

39. Westerman EM, Le Brun PP, Touw DJ, et al. Effect of nebulized colistin sulphate and colistin sulfomethate on lung function in patients with cystic fibrosis: a pilot study. J Cyst Fibros 2004; 3: 23-8

40. McCoy KS. Compounded colistimethate as possible cause of fatal acute respiratory distress syndrome. N Engl J Med 2007; 357: 2310-1

41. Ratjen F, Rietschel E, Kasel D, et al. Pharmacokinetics of inhaled colistin in patients with cystic fibrosis. J Antimicrob Chemother 2006; 57: 306-11

42. Tramper-Stranders GA, Wolfs TF, van Haren Norman S, et al. Controlled trial of cycled antibiotic prophylaxis to prevent initial Pseudomonas aeruginosa infection in children with cystic fibrosis. Thorax 2010; 65: 915-20 
43. Valerius NH, Koch C, Hoiby N. Prevention of chronic Pseudomonas aeruginosa colonisation in cystic fibrosis by early treatment, Lancet 1991; 338: $725-6$

44. Vazquez C, Municio M, Corera M, et al. Early treatment of Pseudomonas aeruginosa colonization in cystic fibrosis. Acta Paediatr 1993; 82: 308-9

45. Hansen CR, Pressler T, Høiby N. Early aggressive eradication therapy for intermittent Pseudomonas aeruginosa airway colonization in cystic fibrosis patients: 15 years experience. J Cyst Fibros 2008; 7: 523-30

46. Taccetti G, Repetto T, Procopio E, et al. Early Pseudomonas aeruginosa colonisation in cystic fibrosis patients. Lancet 2002; 359: 625-6

47. Brochet MS, McDuff AC, Bussières JF, et al. Comparative efficacy of two doses of nebulized colistimethate in the eradication of Pseudomonas aeruginosa in children with cystic fibrosis. Can Respir J 2007; 14: 473-9

48. Jensen T, Pedersen SS, Garne S, et al. Colistin inhalation therapy in cystic fibrosis patients with chronic Pseudomonas aeruginosa lung infection. J Antimicrob Chemother 1987; 19: 831-8

49. Valenza G, Radike K, Schoen C, et al. Resistance to tobramycin and colistin in isolates of Pseudomonas aeruginosa from chronically colonized patients with cystic fibrosis under antimicrobial treatment. Scand J Infect Dis 2010; 42: 885-9

50. Sabuda DM, Laupland K, Pitout J, et al. Utilization of colistin for treatment of multidrug resistant Pseudomonas aeruginosa. Can J Infect Dis Med Microbiol 2008; 19: 413-8

51. Moskowitz SM, Garber E, Chen Y, et al. Colistin susceptibility testing: evaluation of reliability for cystic fibrosis isolates of Pseudomonas aeruginosa and Stenotrophomonas maltophilia. J Antimicrob Chemother 2010; 65: $1416-23$

52. Bauldoff GS, Nunley DR, Manzetti JD, et al. Use of aerosolized colistin sodium in cystic fibrosis patients awaiting lung transplantation. Transplantation 1997; 64: 748-52

53. Dietzsch HJ, Gottschalk B, Heyne K, et al. Cystic fibrosis: comparison of two mucolytic drugs for inhalation treatment (acetylcysteine and arginine hydrochloride). Pediatrics 1975; 55: 96-100

54. Sapienza MA, Kharitonov SA, Horvath I, et al. Effect of inhaled L-arginine on exhaled nitric oxide in normal and asthmatic subjects. Thorax 1998; 53:172-5

55. Gibson RL, Retsch-Bogart GZ, Oermann C, et al. Microbiology, safety, and pharmacokinetics of aztreonam lysinate for inhalation in patients with cystic fibrosis. Pediatr Pulmonol 2006; 41: 656-65

56. Retsch-Bogart GZ, Burns JL, Otto KL, et al. A phase 2 study of aztreonam lysine for inhalation to treat patients with cystic fibrosis and Pseudomonas aeruginosa infection. Pediatr Pulmonol 2008; 43: 47-58

57. Retsch-Bogart GZ, Quittner AL, Gibson RL, et al. Efficacy and safety of inhaled aztreonam lysine for airway Pseudomonas in cystic fibrosis. Chest 2009; 135: 1223-32

58. McCoy KS, Quittner AL, Oermann CM, et al. Inhaled aztreonam lysine for chronic airway Pseudomonas aeruginosa in cystic fibrosis. Am J Respir Crit Care Med 2008; 178: 921-8

59. Oermann CM, Retsch-Bogart GZ, Quittner AL, et al. An 18-month study of the safety and efficacy of repeated courses of inhaled aztreonam lysine in cystic fibrosis. Pediatr Pulmonol 2010; 45: 1121-34

60. Aztreonam for inhalation solution (AZLI) vs tobramycin inhalation solution $\left(\mathrm{TOBI}^{\circledR}\right)$ in patients with $\mathrm{CF}$ and $P$. aeruginosa. http:// clinicaltrials.gov/ct2/show/NCT00757237

61. Hung JC, Hambleton G, Super M. Evaluation of two commercial jet nebulisers and three compressors for the nebulisation of antibiotics. Arch Dis Child 1994; 71: 335-8
62. Adi H, Young PM, Chan HK, et al. Co-spray-dried mannitol-ciprofloxacin dry powder inhaler formulation for cystic fibrosis and chronic obstructive pulmonary disease. Eur J Pharm Sci 2010; 40: 239-47

63. Yang Y, Tsifansky MD, Wu CJ, et al. Inhalable antibiotic delivery using a dry powder co-delivering recombinant deoxyribonuclease and ciprofloxacin for treatment of cystic fibrosis. Pharm Res 2010; 27: 151-60

64. Sweeney LG, Wang Z, Loebenberg R, et al. Spray-freeze-dried liposomal ciprofloxacin powder for inhaled aerosol drug delivery. Int J Pharm 2005; 305: 180-5

65. Bruinenberg P, Otulana B, Blanchard J, et al. The effect of once a day inhaled liposomal ciprofloxacin hydrochloride on sputum bacterial density in cystic fibrosis patients with chronic pulmonary P. aeruginosa colonization. Pediatr Pulmonol 2008; S31: 344

66. Safety and efficacy study of ciprofloxacin for inhalation in patients with noncystic fibrosis bronchiectasis "ORBIT-1". http://clinicaltrials. gov/ct2/show/NCT00889967

67. Sabet M, Miller CE, Nolan TG, et al. Efficacy of aerosol MP-376, a levofloxacin inhalation solution, in models of mouse lung infection due to Pseudomonas aeruginosa. Antimicrob Agents Chemother 2009; 53: 3923 8

68. King P, Lomovskaya O, Griffith DC, et al. In vitro pharmacodynamics of levofloxacin and other aerosolized antibiotics under multiple conditions relevant to chronic pulmonary infection in cystic fibrosis. Antimicrob Agents Chemother 2010; 54: 143-8

69. King P, Citron DM, Griffith DC, et al. Effect of oxygen limitation on the in vitro activity of levofloxacin and other antibiotics administered by the aerosol route against Pseudomonas aeruginosa from cystic fibrosis patients. Diagn Microbiol Infect Dis 2010; 66: 181-6

70. Tsivkovskii R, Sabet M, Tarazi Z, et al. Levofloxacin reduces inflammatory cytokine levels in human bronchial epithelia cells: implications for aerosol MP-376 (levofloxacin solution for inhalation) treatment of chronic pulmonary infections. FEMS Immunol Med Microbiol 2011; 61(2): 141-6

71. Griffith DC, Hansen C, Pressler T, et al. Single dose pharmacokinetics of aerosol MP-376 (levofloxacin solution for inhalation) in cystic fibrosis patients: PK-PD implications. J Cyst Fibros 2008; 7: S26

72. Conrad D, Flume P, Sindel L, et al. Phase 2 b study of inhaled MP-376 (aeroquin, levofloxacin inhalation solution) In stable cystic fibrosis (CF) patients with chronic Pseudomonas aeruginosa (PA) lung infection. Am J Respir Crit Care Med 2010; 181: A2339

73. MP-376 (Aeroquin ${ }^{\mathrm{TM}}$, Levofloxacin for Inhalation) in patients with cystic fibrosis http://clinicaltrials.gov/ct2/show/NCT01180634

74. Registre Français de la Mucoviscidose (RFM) : Bilan des données 2005 de l'Observatoire National de la Mucoviscidose (ONM), Vaincre la Mucoviscidose et Institut National d'Etudes Démographiques (INED), Paris, 2007

75. http://clinicaltrials.gov/ct2/show/NCT01082367

76. http://clinicaltrials.gov/ct2/show/NCT00918957

77. http://clinicaltrials.gov/ct2/show/NCT01316276

78. http://clinicaltrials.gov/ct2/show/NCT01315678

79. http://clinicaltrials.gov/ct2/show/NCT01090908

80. http://clinicaltrials.gov/ct2/show/NCT00645788

81. http://clinicaltrials.gov/ct2/show/NCTQ1270347

Correspondance et offprints : Patrice Vanelle, Laboratoire de PharmacoChimie Radicalaire (LPCR), Faculté de Pharmacie, Universités d'AixMarseille I, II, et III - CNRS, UMR 6264, Laboratoire Chimie Provence, 27 Boulevard Jean Moulin, 13385 Marseille Cedex 05, France.

E-mail : patrice.vanelle@univmed.fr 